\title{
Secondary Hyperparathyroidism in Obese Patients Post Sleeve Gastrectomy
}

\author{
Esraa Altawil ${ }^{1, *}$ \\ Hadeel Alkofide $\mathbb{D}^{2, *}$ \\ Husam Alamri ${ }^{3}$ \\ Noura Alhassan ${ }^{4}$ \\ Hamad Alsubaie ${ }^{3}$ \\ Awadh Alqahtani ${ }^{3}$ \\ Omar Alobaid ${ }^{4}$ \\ 'Pharmacy Department, Clinical \\ Pharmacy Services, King Saud University \\ Medical City, Riyadh, Saudi Arabia; \\ ${ }^{2}$ Department of Clinical Pharmacy, \\ College of Pharmacy, King Saud \\ University, Riyadh, Saudi Arabia; \\ ${ }^{3}$ Department of Surgery, King Saud \\ University, College of Medicine, Riyadh, \\ Saudi Arabia; ${ }^{4}$ Department of Surgery, \\ Colorectal Research Chair, King Saud \\ University, College of Medicine, Riyadh, \\ Saudi Arabia
}

*These authors contributed equally to this work
Correspondence: Omar Alobaid Department of Surgery, Colorectal Research Chair, College of Medicine, King Saud University, P.O. Box 7805, Riyadh,

I I472, Saudi Arabia

Tel +966558670000

Email oalobaid@ksu.edu.sa
Introduction: Bariatric surgery provides an effective option for the management of morbid obesity. Several studies have investigated the association between bariatric surgery and secondary hyperparathyroidism (SHPT). This study aims to compare the levels of blood biomarkers, specifically, calcium (Ca), vitamin D, and phosphate (PO4), and their association with parathyroid hormone (PTH) levels pre- and post-bariatric surgery. In addition, it aims to assess the prevalence of hyperparathyroidism post-bariatric surgery in a tertiary care hospital in Saudi Arabia.

Material and Methods: A prospective cohort study was conducted at a large tertiary care hospital between May 2017 and April 2019. The study included adult obese patients who underwent laparoscopic sleeve gastrectomy (LSG). Patients were excluded if they were known to have any comorbidities, receiving vitamin supplements, and those who had undergone bariatric procedures previously. Routine blood tests, including PTH, vitamin D, Ca, and PO4, were collected at baseline, and post-surgery.

Results: A total of 143 patients who underwent LSG were included in the study. Hyperparathyroidism was observed in $15.4 \%$ of patients at baseline and in $36.4 \%$ of patients' post-surgery $(\mathrm{p}<0.001)$. Low vitamin $\mathrm{D}$ levels, which were highly prevalent before surgery, decreased sustainably (66.4\% pre-operative and $28 \%$ at follow-up after surgery, $\mathrm{P}=0.032$ ). Baseline hypocalcemia was observed in $20.3 \%$ of patients compared to $8.4 \%$ post-surgery $(\mathrm{P}=0.546)$. Hypophosphatemia was present in $60.8 \%$ of subjects at baseline, while the percentage dropped to $21.7 \%$ post-surgery. There was a significant association between PO4 and PTH levels at baseline. Post-operatively, there was a significant correlation between PTH and both vitamin D and calcium levels.

Conclusion: Our study showed that the higher levels of PTH post LSG can be related to vitamin D deficiency and lower calcium levels, despite patients following the provided recommendations for supplementation. This study also emphasizes the importance of routine testing for hyperparathyroidism both before and after bariatric surgery.

Keywords: bariatric surgery, morbid obesity, hyperparathyroidism, calcium, vitamin D

\section{Introduction}

The rising prevalence of obesity represents a serious public health problem, as it is associated with both socio-economic burden and increased personal health risk. ${ }^{1}$ Bariatric surgery provides an effective option for the treatment of morbid obesity as it improves obesity-related comorbidities with sustainable weight reduction. In general, bariatric surgeries are categorized depending on the mechanism used for either restrictive, malabsorptive, or a mix of these two. Recently, laparoscopic sleeve gastrectomy (LSG) has become the most commonly performed among various surgeries. $^{2}$ It is considered to be a restrictive-type procedure, with around 
$80 \%$ of the stomach removed, thus forming a "sleeve" with altered gastric volume and anatomy to reduce the quantities of food intake. ${ }^{2,3}$ Beyond food restriction, LSG is also thought to have a significant metabolic effect. ${ }^{4}$

Following bariatric surgery, nutritional deficiencies are common and include several micronutrients such as calcium, vitamin D, vitamin B12, iron, folic acid, copper, selenium, and zinc. These insufficiencies can lead to several complications affecting bone, blood, and nerves. Although LSG has no intestinal bypass component and preserves passage of food and nutrients through the duodenum and proximal jejunum, which theoretically reduces vitamin and mineral deficiencies, it can ultimately cause nutritional deficiencies due to reduced intake and faster gastric emptying. ${ }^{3}$ Furthermore, obesity per se is well known to be associated with derangement in the levels of some bone minerals, micronutrients and hormones, specifically vitamin D and parathyroid hormone (PTH). ${ }^{5}$ Likewise, weight loss (intended or unintended) has been recognized as a risk factor for bone loss and increases the possibility of hip fractures. ${ }^{6}$

Several studies have investigated the association between bariatric surgery and the development of secondary hyperparathyroidism (SHPT), which is assumed to be a result of calcium malabsorption. Secondary hyperparathyroidism is the leading cause of renal osteodystrophy and bone diseases. The classic and most common osteodystrophy "Osteitis fibrosa cystica" is primarily caused by high bone turnover secondary to uncontrolled high levels of circulating $\mathrm{PTH}^{7,8}$ Indeed, few studies reported a high level of PTH in patients before surgery, which proposed that it could be obesityinduced and not malabsorption related. Furthermore, reduction in bone mineral density (BMD) after surgery has been described in several reports, without an increase in PTH levels, which questions the role of PTH following bariatric surgery and its link to bone loss. ${ }^{9-11}$ This study aims to compare the levels of blood biomarkers, specifically, Ca, vitamin D, and phosphate (PO4), and their association with parathyroid hormone (PTH) levels in pre- and post-bariatric surgery. In addition, it aims to assess the prevalence of hyperparathyroidism post-bariatric surgery in a tertiary care hospital in Saudi Arabia. To the best of our knowledge, this is the only national study available to date to investigate such an association.

\section{Materials and Methods}

\section{Study Design and Setting}

A prospective cohort study was conducted at a tertiarycare teaching hospital, located in Riyadh, Saudi Arabia.
Data were collected from May 2017 to April 2019. Approval of the study protocol was obtained from the King Saud University Medical City (KSUMC) Institutional Review Board (IRB) before the commencement of the study, and it was conducted in accordance with the Declaration of Helsinki.

\section{Patients' Selection}

The study included adult (age $\geq 18$ years) obese patients $\left(\mathrm{BMI} \geq 30 \mathrm{~kg} / \mathrm{m}^{2}\right)$ undergoing laparoscopic sleeve gastrectomy (LSG). Patients were excluded if they were known to have any comorbidities (eg, diabetes mellitus, hypertension, hypercholesterolemia, or hypertriglyceridemia), using medications to treat chronic conditions, receiving any vitamin supplements or herbal product, and patients who had undergone bariatric surgery previously.

\section{Data Collection}

All patients admitted for elective LSG were screened for eligibility. The research team met patients, providing them with a thorough explanation of the study's goals and objectives. In an agreement to be enrolled in the study, patients were asked to sign an informed consent form.

Routine blood tests including complete blood count (CBC), serum albumin, total protein, hemoglobin, parathyroid hormone $(\mathrm{PTH})$, vitamin $\mathrm{D}$, corrected calcium (Ca), phosphate (PO4), renal profile, and bone quality measurement tests were collected pre-operatively and during follow-up visits at 12 to 18 months post-surgery. The normal PTH serum concentration range was defined from 1.6 to $6.9 \mathrm{pmol} / \mathrm{L}$, normal 25-hydroxy vitamin D concentrations from 50 to $250 \mathrm{nmol} / \mathrm{L}$, normal calcium levels from 2.2 to $2.7 \mathrm{mmol} / \mathrm{L}$, and normal phosphate levels from 1.1 to $1.45 \mathrm{mmol} / \mathrm{L}$.

Post-operatively, all patients were instructed to take the following as per standard local practice guidelines: 1) multivitamin tablets $\left(\right.$ Centrum $^{\mathbb{B}}$ ) one tablet daily, 2) Neurobion ${ }^{\circledR}$ (Vitamin B1, B6, B12) one tablet daily, 3) Calcium-vitamin D (500 mg/400 IU) one tablet daily, 4) Ferrous fumarate $200 \mathrm{mg}$ one tablet twice daily, and 5) biotin $10,000 \mu \mathrm{g}$ one tablet daily starting after surgery and continued for one year. Compliance with medications was addressed in the clinic and patients were counselled during their visits regarding medication intake. Although we do not have data on subject's adherence, it was assessed by a clinical pharmacist in routine care in the bariatric clinic. If any compliance issues were seen, the subjects would be counselled on proper medication use. 


\section{Statistical Analysis}

Data were analyzed by using Statistical Package for Social Studies (SPSS 22; IBM Corp., New York, NY, USA) and $\mathrm{R}$ version 4.0.3. Continuous variables were expressed as mean \pm standard deviation, and categorical variables were expressed as percentages. Paired $t$-test was used to compare continuous variables, and chi-square test was used to compare categorical variables, pre- and post-operatively. Pearson correlation coefficient was used to assess the relationship between (vitamin $\mathrm{D}, \mathrm{Ca}, \mathrm{PO} 4)$ and $\mathrm{PTH}$ level. Shapiro-Wilk test was used to evaluate the normality of the data. A p-value $<0.05$ was considered statistically significant.

Univariate and multivariate linear regression models were used to assess the relationship between PTH levels pre- and post-operatively and relevant clinical characteristics including age, gender, weight, and $\mathrm{Ca}, \mathrm{PO} 4$ and vitamin D levels. Variables associated with PTH levels in univariate analysis at a p-value of less than 0.1 were included in multivariate models. Univariate and multivariate logistics regression analysis was used to investigate the association between clinical characteristics and SHPT status pre- and post-operatively. Variables associated with PTH levels in univariate analysis at a p-value of less than 0.1 were included in multivariate logistic analysis. In this study, SHPT was defined as PTH levels above 6.9 $\mathrm{pmol} / \mathrm{L}$.

\section{Results}

A total of 143 patients who underwent LSG and met the study inclusion criteria were included in the study. All subjects had data available at baseline (pre-operatively) and 12-18 months follow-up (post-operatively). The mean age was $31.30 \pm 9.70$ years, with the majority being females $(58 \%)$. The pre-operative mean BMI was $44.95 \pm 8.05 \mathrm{~kg} / \mathrm{m}^{2}$. Anthropometric and laboratory variables before and after the surgery for all patients are listed in Table 1.

Hyperparathyroidism (ie, high PTH levels) was observed in $15.4 \%$ of patients at baseline and in $36.4 \%$ post-surgery $(\mathrm{P}<0.001)$. Low vitamin $\mathrm{D}$ levels, which were highly prevalent before surgery, decreased significantly (66.4\% pre-operative and $28.0 \%$ at follow-up after surgery, $\mathrm{P}=0.032$ ). Baseline hypocalcemia was seen in $20.3 \%$ of patients compared to $8.4 \%$ post-surgery $(\mathrm{P}=$ 0.546). Hypophosphatemia occurred in $60.8 \%$ of subjects, and this number dropped to 31 patients $(21.7 \%)$ postsurgery (Table 2).

Pre-operatively, only baseline PO4 level was associated with PTH levels in univariate linear regression analysis (Beta coefficient $=-2.61$, confidence interval $[\mathrm{CI}]=-4.49,-0.73)$. This association remained significant in multivariate analysis. Post-operatively, there was a significant correlation between PTH and both Ca (Beta coefficient $=-4.75$, confidence interval $[\mathrm{CI}]=-8.36$, -1.14 ), and vitamin D levels (Beta coefficient $=-0.02$, $\mathrm{CI}=-0.03,-0.01)$. These relationships remained significant in multivariate analysis (Table 3).

Patients were categorized into four groups according to their laboratory values and status before or after the surgery. Group 1 consists of patients with SHPT pre-surgery, group 2 includes patients without SHPT pre-surgery, group 3 includes patients with SHPT post-surgery, and group 4 includes patients without SHPT post-surgery. After surgery, all groups had maintained normal phosphate-metabolism homeostasis. There was no statistically significant difference in vitamin D levels between patients with SHPT and patients without SHPT pre-operatively. In contrast, a statistically significant increase in vitamin D levels was observed post-operatively, and this effect was predominant in patients without SHPT $(\mathrm{P}=0.011)$. Calcium levels were higher in patients with SHPT compared to patients without SHPT pre-operatively, but the difference did not reach a significant level. Post-operatively, calcium levels were significantly lower in patients with SHPT than in patients without SHPT (Table 4).

When analyzing the relationship between high PTH levels (ie, SHPT) and clinical characteristics using logistic regression, none of the covariates were significantly associated with SHPT pre-operatively. Post-operatively, $\mathrm{Ca}$ $(\mathrm{OR}=0.00, \mathrm{CI}=0.00,0.10), \mathrm{PO}(\mathrm{OR}=11.1 \mathrm{CI}=1.22$, $128)$, and vitamin $\mathrm{D}(\mathrm{OR}=0.99, \mathrm{CI}=0.97,1.00)$ were significantly associated with SHPT in univariate analysis. On the other hand, only the relationship with $\mathrm{Ca}$ level remained significant in multivariate analysis (Table 5).

\section{Discussion}

The most remarkable finding in our study is that $15 \%$ of the studied patients already had SHPT before surgery. Previous reports have proposed that vitamin D deficiency is the main reason behind the development of SHPT and is commonly seen in obese patients. It has been reported that the incidence of vitamin D deficiency in bariatric patients can reach up to $96 \% .^{12,13}$ It is postulated that the high 
Table I Patients Demographic, Anthropometric, and Laboratory Values Pre- and Postoperative

\begin{tabular}{|c|c|c|c|}
\hline Variables & Baseline $n=143$ & Postoperative $n=\mid 43$ & p-Value \\
\hline Age (years) - mean \pm SD & $31.30 \pm 9.70$ & - & - \\
\hline \multicolumn{4}{|l|}{ Gender - n (\%) } \\
\hline Male & $60(42.0 \%)$ & - & - \\
\hline Female & $83(58.0 \%)$ & - & - \\
\hline Height $(\mathrm{cm})$ - mean \pm SD & $164.40 \pm 9.30$ & - & - \\
\hline Weight $(\mathrm{kg})$ - mean \pm SD & $121.56 \pm 26.36$ & $86.63 \pm 20.66$ & $0.001 *$ \\
\hline BMI $\left(\mathrm{kg} / \mathrm{m}^{2}\right)-$ mean $\pm S D$ & $44.95 \pm 8.05$ & $31.80 \pm 6.42$ & $0.001 *$ \\
\hline $\mathrm{Hgb}(\mathrm{gm} / \mathrm{L})-$ mean $\pm \mathrm{SD}$ & $134.07 \pm 16.35$ & $135.60 \pm 14.04$ & 0.068 \\
\hline$M C V(f L)$ - mean $\pm S D$ & $80.54 \pm 6.58$ & $81.84 \pm 7.23$ & $0.030 *$ \\
\hline INR (seconds) - mean \pm SD & $1.05 \pm 0.23$ & $1.02 \pm 0.08$ & 0.538 \\
\hline APTT (seconds) - mean \pm SD & $34.44 \pm 7.78$ & $36.28 \pm 4.22$ & $0.006 *$ \\
\hline $\mathrm{Ca}(\mathrm{mmol} / \mathrm{L})-$ mean $\pm \mathrm{SD}$ & $2.29 \pm 0.15$ & $2.33 \pm 0.10$ & $0.001 *$ \\
\hline PO4 (mmol/L) - mean \pm SD & $1.07 \pm 0.18$ & $1.2 \pm 0.17$ & $0.001 *$ \\
\hline PTH (pmol/L) - mean \pm SD & $5.08 \pm 2.04$ & $6.13 \pm 2.41$ & $0.001 *$ \\
\hline Total protein (gm/L) - mean \pm SD & $71.66 \pm 7.39$ & $70.40 \pm 8.03$ & 0.139 \\
\hline Albumin (gm/L) - mean \pm SD & $36.68 \pm 19.69$ & $36.85 \pm 4.81$ & 0.920 \\
\hline Vitamin $D(\mathrm{nmol} / \mathrm{L})$ - mean \pm SD & $47.38 \pm 30.93$ & $71.49 \pm 31.76$ & $0.001 *$ \\
\hline $\operatorname{SrCr}(\mu \mathrm{mol} / \mathrm{l})-$ mean $\pm \mathrm{SD}$ & $61.94 \pm 13.16$ & $59.42 \pm 13.20$ & $0.019 *$ \\
\hline
\end{tabular}

Note: *Statistical significance was set at the level of $P$ value $<0.05$.

Abbreviations: APTT, activated partial thromboplastin time; BMI, body mass index; Ca, calcium; Hgb, hemoglobin; INR, international normalized ratio; MCV, mean corpuscular volume; PO4, phosphate; PTH, parathyroid hormone; SD, standard deviation; SrCr, serum creatinine.

Table 2 Level of Laboratory Values (Ca, PO4, PTH, Vitamin D) Pre and Post Surgery

\begin{tabular}{|c|c|c|c|c|c|c|}
\hline \multirow[t]{2}{*}{ Parameter } & \multirow[t]{2}{*}{ Levels } & \multicolumn{2}{|c|}{ Baseline } & \multicolumn{2}{|c|}{ Post-Operative } & \multirow[t]{2}{*}{ p-value } \\
\hline & & $\mathbf{n}$ & $\%$ & $\mathbf{n}$ & $\%$ & \\
\hline \multirow[t]{2}{*}{$\mathrm{Ca}(2.2-2.7 \mathrm{mmol} / \mathrm{L})$} & Normal & 114 & 79.7 & $|3|$ & 91.6 & 0.546 \\
\hline & Low & 29 & 20.3 & 12 & 8.4 & \\
\hline \multirow[t]{3}{*}{ PO4 (I.I-I.45 mmol/L) } & Normal & 51 & 35.7 & 106 & 74.1 & $0.038 *$ \\
\hline & Low & 87 & 60.8 & 31 & 21.7 & \\
\hline & High & 5 & 3.5 & 6 & 4.2 & \\
\hline \multirow[t]{2}{*}{ PTH (I.6-6.9 pmol/L) } & Normal & 121 & 84.6 & 91 & 63.6 & $0.001 *$ \\
\hline & High & 22 & 15.4 & 52 & 36.4 & \\
\hline \multirow[t]{2}{*}{ Vitamin D (50-250 nmol/L) } & Normal & 48 & 33.6 & 103 & 72.0 & $0.032 *$ \\
\hline & Low & 95 & 66.4 & 40 & 28.0 & \\
\hline
\end{tabular}

Note: *Statistical significance was set at the level of $\mathrm{P}$ value $<0.05$.

Abbreviations: Ca, calcium; PO4, phosphate; PTH, parathyroid hormone.

incidence of vitamin D deficiency in obese individuals is a consequence of increased uptake of the vitamin by adipose tissue, in addition to limited physical activity, further limited sun exposure and affected hepatic synthesis of vitamin $\mathrm{D}$ due to impaired liver function caused by hepatic steatosis. ${ }^{14}$ In our study, pre-operative PTH levels 
Table 3 Association Between PTH Level and Clinical Characteristics Pre- and Post-Operatively Using Linear Regression Analysis

\begin{tabular}{|c|c|c|c|c|c|c|}
\hline \multirow[t]{2}{*}{ Variables } & \multicolumn{3}{|c|}{ Univariate Analysis } & \multicolumn{3}{|c|}{ Multivariate Analysis } \\
\hline & $\mathbf{B}$ & $95 \% \mathrm{Cl}$ & p-Value & $\boldsymbol{\beta}$ & $95 \% \mathrm{Cl}$ & p-Value \\
\hline \multicolumn{7}{|c|}{ Pre-operatively } \\
\hline Age & 0.08 & $-0.6-0.76$ & 0.8 & - & - & - \\
\hline Gender & 0.03 & $-0.01,0.06$ & 0.2 & - & - & - \\
\hline Weight & 0.00 & $-0.01,0.02$ & 0.5 & - & - & - \\
\hline $\mathrm{Ca}$ & 0.85 & $-1.42,3.13$ & 0.5 & - & - & - \\
\hline $\mathrm{PO} 4$ & -2.61 & $-4.49,-0.73$ & $0.007^{*}$ & -2.59 & $-4.46,-0.72$ & $0.007^{*}$ \\
\hline Vitamin D & -0.01 & $-0.02,0.00$ & 0.089 & -0.01 & $-0.02,0.00$ & 0.089 \\
\hline \multicolumn{7}{|c|}{ Post-operatively } \\
\hline Age & 0.03 & $-0.01,0.07$ & 0.2 & - & - & - \\
\hline Gender & -0.25 & $-1.06,0.56$ & 0.5 & - & - & - \\
\hline Weight & -0.01 & $-0.03,0.01$ & 0.6 & - & - & - \\
\hline $\mathrm{Ca}$ & -6.52 & $-10.2,-2.85$ & $<0.00$ I* & -4.75 & $-8.36,-1.14$ & $0.010 *$ \\
\hline $\mathrm{PO} 4$ & 2.04 & $-0.26,4.34$ & $0.08 I$ & 1.90 & $-0.24,4.05$ & $0.08 I$ \\
\hline Vitamin D & -0.02 & $-0.04,-0.01$ & $<0.00 I^{*}$ & -0.02 & $-0.03,-0.01$ & $<0.00$ I* \\
\hline
\end{tabular}

Note: *Statistical significance was set at the level of $\mathrm{P}$ value $<0.05$.

Abbreviations: $\beta$, beta coefficient; $\mathrm{Ca}$, calcium; $\mathrm{Cl}$, confidence interval; $\mathrm{PO}$, phosphate; PTH, parathyroid.

Table 4 Comparison of Pre- and Post-Operative Vitamin D, Ca, and PO4 Levels by SHPT Status

\begin{tabular}{|l|c|c|c|c|c|c|}
\hline \multirow{2}{*}{ Parameter } & \multicolumn{3}{|c|}{ Pre-Operative } & \multicolumn{3}{c|}{ Post-Operative } \\
\cline { 2 - 7 } & With SHPT N=22 & Without SHPT N= 121 & p-Value & With SHPT N= 52 & Without SHPT N=91 & p-Value \\
\hline Vitamin D - mean \pm SD & $39.13 \pm 20.43$ & $48.89 \pm 32.32$ & 0.174 & $63.35 \pm 24.35$ & $76.15 \pm 34.57$ & $0.011 *$ \\
Ca - mean \pm SD & $2.33 \pm 0.21$ & $2.28 \pm 0.13$ & 0.151 & $2.29 \pm 0.11$ & $2.35 \pm 0.09$ & $0.001 *$ \\
PO4 - mean \pm SD & $1.02 \pm 0.19$ & $1.08 \pm 0.17$ & 0.133 & $1.24 \pm 0.13$ & $1.18 \pm 0.19$ & 0.077 \\
\hline
\end{tabular}

Note: *Statistical significance was set at the level of $P$ value $<0.05$.

Abbreviations: $\mathrm{Ca}$, calcium; PO4, phosphate; SD, standard deviation; SHPT, secondary hyperparathyroidism.

correlated only with phosphate levels on univariate analysis; however, $66.4 \%$ of our patients had pre-operative vitamin D deficiency.

Laparoscopic sleeve gastrectomy is reported to have lower rates of SHPT and vitamin D deficiency when compared to malabsorptive procedures, such as Roux-en-Y gastric bypass, one anastomosis gastric bypass and duodenal switch. ${ }^{11,12,15}$ LSG can affect calcium uptake indirectly through reduced volume and decreased gastric acidity. The patients in our study presented with an increased rate of secondary hyperparathyroidism from $15.4 \%$ preoperatively to $36.4 \%$ post-operatively, and both vitamin $\mathrm{D}$ and calcium appeared to be the main contributors. As seen in the results of the linear regression models, PTH levels were associated with vitamin D and calcium levels, specifically post-operatively. Moreover, the results of the logistic regression model showed a strong relationship between SHPT status and calcium levels, and with a lower extent of vitamin D levels. However, the association between vitamin D levels and SHPT status did not reach statistical significance in the multivariable analysis $(p=0.062)$. Changes in vitamin D levels and consequently calcium absorption provoke PTH production and SHPT and increased PTH affects the mobilization of the skeletal calcium, aiming to maintain a state of normocalcemia. Nevertheless, the increased rate of secondary hyperparathyroidism can be affected by other unmeasured variables, such as Fasting Blood Sugar (FBS), HbAlc, and cholesterol.

The results of previous reports support some of our findings observed. For instance, a cohort study of 120 patients who underwent several bariatric surgeries found that the postoperative hyperparathyroidism was significantly linked to calcium and vitamin D deficiencies and less weight reduction. In addition, this study shows that sleeve gastrectomy is associated with a significantly lower 
Table 5 Association Between SHPT Status and Clinical Characteristics Pre- and Post-Operatively Using Logistic Regression Analysis

\begin{tabular}{|c|c|c|c|c|c|c|}
\hline \multirow[t]{2}{*}{ Variables } & \multicolumn{3}{|c|}{ Univariate Analysis } & \multicolumn{3}{|c|}{ Multivariate Analysis } \\
\hline & OR & $95 \% \mathrm{Cl}$ & p-Value & OR & $95 \% \mathrm{Cl}$ & p-Value \\
\hline \multicolumn{7}{|c|}{ Pre-operatively } \\
\hline Age & 1.00 & $0.96,1.05$ & 0.87 & - & - & - \\
\hline Gender & 1.05 & $0.42,2.73$ & 0.91 & - & - & - \\
\hline Weight & 1.00 & $0.99,1.02$ & 0.72 & - & - & - \\
\hline $\mathrm{Ca}$ & 5.97 & $0.31,115$ & 0.23 & - & - & - \\
\hline $\mathrm{PO} 4$ & 0.11 & $0.01,1.74$ & 0.12 & - & - & - \\
\hline Vitamin D & 0.99 & $0.96,1.00$ & 0.13 & - & - & - \\
\hline \multicolumn{7}{|c|}{ Post-operatively } \\
\hline Age & 1.02 & $0.99,1.06$ & 0.25 & - & - & - \\
\hline Gender & 0.86 & $0.43,1.73$ & 0.68 & - & - & - \\
\hline Weight & 0.99 & $0.98,1.01$ & 0.42 & - & - & - \\
\hline $\mathrm{Ca}$ & 0.00 & $0.00,0.10$ & $0.00 I^{*}$ & 0.01 & $0.00,0.28$ & $0.010^{*}$ \\
\hline $\mathrm{PO} 4$ & 11.1 & $1.22,128$ & 0.032 & 10.4 & $|.05| 4 \mid$, & 0.060 \\
\hline Vitamin D & 0.99 & $0.97,1.00$ & $0.017^{*}$ & 0.99 & $0.97,1.00$ & 0.062 \\
\hline
\end{tabular}

Note: *Statistical significance was set at the level of $P$ value $<0.05$.

Abbreviations: $\mathrm{Ca}$, calcium; $\mathrm{Cl}$, confidence interval; OR, odds ratio; PO4, phosphate; SHPT, secondary hyperparathyroidism.

number of hyperparathyroidism cases. ${ }^{11}$ A retrospective observational study of a small group of patients was published in 2012 investigating the consequences of sleeve gastrectomy on several parameters, such as calcium, vitamin D, and PTH. This study reported a constant drop in PTH and increase in vitamin D levels for up to two years post LSG. ${ }^{16}$ Another observational study by Gehrer et al, which was a prospective cohort, compared micronutrient and vitamin deficiencies in patients undergoing several bariatric surgeries pre- and post-operatively. Overall 136 patients were studied (50 patients underwent LSG). Postoperatively in the LSG group, $14 \%$ of patients had increased $\mathrm{PTH}$, and $32 \%$ had vitamin D insufficiency. The highest incidence of hyperparathyroidism was observed at six months post-surgery; afterwards, the incidence decreased. On the other hand, the rate of vitamin D deficiency increased to the highest at 1-year postsurgery and then decreased back at two years. ${ }^{17}$ Conversely, a study of 40 patients 5 years post-sleeve gastrectomy reported a significant increase in the rate of SHPT from $17.5 \%$ pre-operatively to $60.7 \%$ at 5 years, ${ }^{18}$ with calcium deficiency appearing to be the main reason. This increase was also observed in a recent study of 390 patients including $132 \mathrm{LSG}$, reporting increasing rates of SHPT up to $45.5 \%$ in patients post LSG 2-years postoperatively. ${ }^{19}$ Interestingly, in a retrospective cohort study of 226 morbidly obese patients who underwent
Roux-en-Y gastric bypass on vertical-banded gastroplasty, $89 \%$ of patients diagnosed with presurgical secondary normocalcemic hyperparathyroidism (SNHPT), with $24 \%$ of these patients recovered within 18 months after surgery. Even though they recovered from this disorder after surgery, their serum PTH levels were statistically significantly $(p<0.05)$ higher than those of other patients throughout the study follow-up period. ${ }^{20}$ These variable findings can be explained by differences in dietary supplement protocols and whether or not additional supplemental calcium and vitamin D were provided during follow-up. Current guidelines from the American Society for Metabolic and Bariatric Surgery recommend routine post-operative micronutrient supplementation. ${ }^{21}$ However, the optimal duration of this supplementation is not well defined, and experts usually suggest continuous nutritional follow-up of all bariatric patients. Our findings further support the need for long-term nutritional monitoring and active adjustment of dietary supplement needs.

Although our study provides a vital insight into the relationship between SHPT and LSG, there are several limitations. First, this was an observational study; however, data were collected prospectively with no missing values. Furthermore, the observational nature of this study makes it difficult to account for unmeasured confounders, which might explain some of the relationships seen in the regression analyses. Second, while all subjects were 
prescribed supplements, whether they adhered to their use or not is unknown. Finally, this was a single-center study of healthy young patients with no major comorbidities, which might affect the generalizability of the results. Nevertheless, we included a large sample of subjects who underwent LSG, and to our knowledge, this is the first local study to describe the rates of SHPT pre- and post-LSG.

\section{Conclusion}

Our study showed a high rate of SHPT in patients undergoing LSG, which can be attributed to changes in vitamin $\mathrm{D}$ and calcium levels. Moreover, the study findings emphasize the importance of routine testing for hyperparathyroidism both before and after LSG. An optimal regimen of calcium and vitamin D supplementations needs to be specified, and this would be an important area for further research.

\section{Data Sharing Statement}

All data analyzed during this study are included in this published article.

\section{Acknowledgment}

The authors are grateful to the Saudi Laparoscopic Society for funding the research.

\section{Disclosure}

The authors report no conflicts of interest in this work.

\section{References}

1. Bluher M. Obesity: global epidemiology and pathogenesis. Nat Rev Endocrinol. 2019;15(5):288-298. doi:10.1038/s41574-019-0176-8

2. Angrisani L, Santonicola A, Iovino P, et al. Bariatric surgery worldwide 2013. Obes Surg. 2015;25(10):1822-1832. doi:10.1007/s11695$015-1657-z$

3. Gjessing H, Nielsen H, Mellgren G, et al. Energy intake, nutritional status and weight reduction in patients one year after laparoscopic sleeve gastrectomy. Springerplus. 2013;2(1):352. doi:10.1186/21931801-2-352

4. Sandoval D. Bariatric surgeries: beyond restriction and malabsorption. Int J Obes. 2011;35(Suppl 3):S45-49. doi:10.1038/ijo.2011.148

5. Mussolino ME, Looker AC, Madans JH, et al. Risk factors for hip fracture in white men: the NHANES I epidemiologic follow-up study. J Bone Miner Res. 1998;13(6):918-924. doi:10.1359/jbmr.1998.13.6.918

6. Ensrud K, Fullman R, Barrett-Connor E, et al. Voluntary weight reduction in older men increases hip bone loss: the osteoporotic fractures in men study. $J$ Clin Endocrinol Metab. 2005;90 (4):1998-2004. doi:10.1210/jc.2004-1805
7. Hewitt S, Sovik T, Aasheim E, et al. Secondary hyperparathyroidism, vitamin $\mathrm{D}$ sufficiency, and serum calcium 5 years after gastric bypass and duodenal switch. Obes Surg. 2013;23(3):384-390. doi:10.1007/ s11695-012-0772-3

8. Grethen E, McClintock R, Gupta C, et al. Vitamin D and hyperparathyroidism in obesity. $J$ Clin Endocrinol Metab. 2011;96 (5):1320-1326. doi:10.1210/jc.2010-2202

9. Vilarrasa N, Vilarrasa N, Gordejuela A, et al. Effect of bariatric surgery on bone mineral density: comparison of gastric bypass and sleeve gastrectomy. Obes Surg. 2013;23(12):2086-2091. doi:10.1007/s11695-013-1016-x

10. Bredella M, Greenblatt L, Eajazi A, et al. Effects of Roux-en-Y gastric bypass and sleeve gastrectomy on bone mineral density and marrow adipose tissue. Bone. 2017;95:85-90. doi:10.1016/j. bone.2016.11.014

11. Jalali S, Azadbakht M, Azadbakht S, et al. Prevalence of secondary hyperparathyroidism following bariatric surgery. Int J Surg Open. 2020;27:214e219. doi:10.1016/j.ijso.2020.11.022

12. Vix M, Liu K, Diana M, et al. Impact of Roux-en-Y gastric bypass versus sleeve gastrectomy on vitamin D metabolism: short-term results from a prospective randomized clinical trial. Surg Endosc. 2014;28(3):821-826. doi:10.1007/s00464-013-3276-x

13. Ducloux R, Nobécourt E, Chevallier J, et al. Vitamin D deficiency before bariatric surgery: should supplement intake be routinely prescribed? Obes Surg. 2011;21(5):556-560. doi:10.1007/s11695010-0352-3

14. Wortsman J, Matsuoka L, Chen T, et al. Decreased bioavailability of vitamin D in obesity. Am J Clin Nutr. 2000;72(3):690-693. doi:10.1093/ajen/72.3.690

15. Wei J, Lee W, Chong K, et al. High incidence of secondary hyperparathyroidism in bariatric patients: comparing different procedures. Obes Surg. 2018;28(3):798-804. doi:10.1007/s11695-017-2932-y

16. Ruiz-Tovar J, Oller I, Tomas A, et al. Mid-term effects of sleeve gastrectomy on calcium metabolism parameters, vitamin D and parathormone (PTH) in morbid obese women. Obes Surg. 2012;22 (5):797-801. doi:10.1007/s11695-011-0582-z

17. Gehrer S, Kern B, Peters T, et al. Fewer nutrient deficiencies after laparoscopic sleeve gastrectomy (LSG) than after laparoscopic Roux-Y-gastric bypass (LRYGB)-a prospective study. Obes Surg. 2010;20(4):447-453. doi:10.1007/s11695-009-0068-4

18. Chou J, Lee W, Almalki O, et al. Dietary intake and weight changes 5 years after laparoscopic sleeve gastrectomy. Obes Surg. 2017;27 (12):3240-3246. doi:10.1007/s11695-017-2765-8

19. Misra S, Balasubramanian S, Isaac U, et al. Is the current micronutrient supplementation adequate in preventing deficiencies in Indian patients? Short- and mid-term comparison of sleeve gastrectomy and roux-en-y gastric bypass. Obes Surg. 2020;30(9):3480-3488. doi:10.1007/s11695-020-04674-2

20. Casella C, Molfino S, Mittempergher F, et al. Predictive factors of secondary normocalcemic hyperparathyroidism after Roux-en-Y gastric bypass. Int $J$ Endocrinol. 2018;2018:1-8. doi:10.1155/2018/ 5010287

21. Mechanick J, Apovian C, Brethauer S, et al. Clinical practice guidelines for the perioperative nutrition, metabolic, and nonsurgical support of patients undergoing bariatric procedures - 2019 update: cosponsored by American Association of Clinical Endocrinologists/ American College of Endocrinology, the Obesity Society, American Society for Metabolic \& Bariatric Surgery, Obesity Medicine Association, and American Society of Anesthesiologists - Executive summary. Endocr Pract. 2019;25:1346-1359. doi:10.4158/GL-20190406 


\section{Publish your work in this journal}

Diabetes, Metabolic Syndrome and Obesity: Targets and Therapy is an international, peer-reviewed open-access journal committed to the rapid publication of the latest laboratory and clinical findings in the fields of diabetes, metabolic syndrome and obesity research. Original research, review, case reports, hypothesis formation, expert opinion and commentaries are all considered for publication. The manuscript management system is completely online and includes a very quick and fair peer-review system, which is all easy to use. Visit http://www.dovepress.com/testimonials.php to read real quotes from published authors.

Submit your manuscript here: https:/www.dovepress.com/diabetes-metabolic-syndrome-and-obesity-targets-and-therapy-journal 